\title{
Comparison of Ultrasonography and Conventional Fluoroscopy Guided Caudal Epidural Injection in Chronic Low Back Pain
}

\author{
Serkan SENKAL ${ }^{1}$, Ender SIR ${ }^{2}$ \\ ${ }^{1}$ University of Health Sciences, Gulhane Medical Faculty, Department of Anesthesiology and Reanimation, Ankara, Turkey \\ ${ }^{2}$ Gulhane Training and Research Hospital, Department of Algology and Pain Medicine, Ankara, Turkey \\ Corresponding author: Ender SIR endersir@gmail.com
}

\section{ABSTRACT}

AIM: To compare the efficacies of fluoroscopy- and ultrasound (US)-guided caudal epidural steroid injections (CESIs) in patients with chronic low back pain (LBP).

MATERIAL and METHODS: This study included patients with chronic LBP who underwent US- (Group U; n=90) or fluoroscopyguided (Group F; $n=90$ ) CESI. The procedure time, successful injection rate on the first attempt, complication rate, Oswestry Disability Index (ODI) score, and Numeric Rating Scale (NRS) score before CESI and after 3 weeks and 3 months of CESI were analyzed.

RESULTS: NRS and ODI scores improved at 3 weeks $(p<0.001)$ and 3 months $(p<0.001)$ after CESls. No significant differences were noted between the two groups for the NRS $(p=0.22$ and $p=0.47)$ and ODI $(p=0.58, p=0.22)$ scores. Moreover, the CESI procedure time was significantly shorter $(p<0.001)$ and the successful injection rate on the first attempt was significantly higher $(p=0.002)$ in Group $U$ than in Group F. The complication rate difference was statistically insignificant between the two groups ( $p>0.05$ ).

CONCLUSION: Outcomes of US-guided CESI were superior than those of fluoroscopy-guided CESI considering the successful injection rate on the first attempt and procedure time. In addition, US-guided CESI was as effective as fluoroscopy-guided CESI and did not expose patients and practitioners to radiation.

KEYWORDS: Caudal anesthesia, Epidural injections, Fluoroscopy, Interventional ultrasonography, Low back pain

ABBREVIATIONS: BMI: Body mass Index, BS: Base of sacrum, CES: Caudal epidural space, CESI: Caudal epidural steroid injection, CSF: Cerebrospinal fluid, LBP: Low back pain, N: Needle, NRS: Numeric rating scale, ODI: Oswestry disability Index, SH: Sacral horn, US: Ultrasound

\section{INTRODUCTION}

L ow back pain (LBP) is the most frequent musculoskeletal complaint, with an incidence of $5 \%-30 \%$ and a prevalence of $60 \%-90 \%$ in the lifetime (2). Generally, LBP is caused by mechanical compression of an intervertebral herniated disc and related inflammation. Irritation and inflammation of spinal nerve roots present with LBP radiating down the buttock or leg (18). Initial treatments are generally conservative, such as rest, physical therapy, and use of nonsteroidal anti-inflammatory drugs and opioids $(6,9)$. However, interventional pain management therapies such as epidural steroid injections can also be administered (5). The treatment mechanism of epidural steroid injection is the reduction of inflammation and irritation caused by mechanical compression of spinal nerve roots (14).

An epidural injection can be administered through three approaches: interlaminar, transforaminal, and caudal approaches $(4,12)$. Although transforaminal anterior epidural steroid injection is more targeted, caudal epidural steroid injection (CESI) has advantages in terms of complications 
and ease of administration (16). For decades, CESls were performed blind or under fluoroscopy guidance (13). However, the interest in fluoroscopy-guided blocks reduced because of radiation exposure to both patients and physicians (15). Recently, ultrasound (US) guidance has gained popularity in CESIs (1).

In the current study, the efficacies of US- and fluoroscopyguided CESls were compared for the procedure time, successful injection rate on the first attempt, complication rate, improvement in pain level, and functional capacity of the patients.

\section{MATERIAL and METHODS}

This study was approved by the Institutional Review Board [University of Health Sciences, Gulhane Medical Faculty Institutional Ethical Committee (2019/5, 19/100)]. We analyzed the follow-up forms of patients who were admitted to the Algology and Pain Medicine Department between September 2016 and January 2019 with the diagnosis of chronic LBP and were treated with US-guided or fluoroscopy-guided CESI. Inclusion criteria were as follows: age 18-85 years, LBP for more than 3 months, and unresponsiveness to conservative treatments such as non-steroidal anti-inflammatory drugs, muscle relaxant drugs, and physical therapy. Exclusion criteria were as follows: presence of a local or systemic infection, missing follow-up data, and history of allergy to contrast, local anesthetic drugs, and coagulopathy.

All procedures were performed in the operating room. The patients were placed in the prone position by placing a cushion beneath the abdomen for optimal imaging. We performed standard noninvasive monitoring of blood pressure, electrocardiography, and pulse oximetry and established an intravenous access. Asepsis of the caudal region was performed using chlorhexidine.

In the US-guided group (Group U), a high-frequency linear US probe (HFL50xp, 15-6 MHz) of an US machine (Edge, Sonosite, Bothell, WA, USA) was used to identify the sacral hiatus in the out-of-plane approach (Figure 1A). Thereafter, the US probe was rotated $90^{\circ}$, brought to the in-plane position, and the sacral canal was observed. CESI was not performed in patients with anomalies in caudal structures. After infiltration with $1-3 \mathrm{~mL}$ of $2 \%$ lidocaine, a $22-\mathrm{G} 100-$ mm echogenic needle (Echoplex ${ }^{\odot}$, Vygon, Ecouen, France) was advanced from the sacrococcygeal membrane to the caudal epidural space under real-time US guidance (Figure 1B). The presence of blood and cerebrospinal fluid (CSF) were determined by negative pressure aspiration using the needle. The location of the epidural area was confirmed by observing the injection site using color Doppler and expansion of the epidural area (Figure 1C). A mixture of $2 \%$ lidocaine $(2 \mathrm{~mL})$ and $8 \mathrm{mg}$ dexamethasone $(2 \mathrm{~mL})$ in saline (total volume: 10 $\mathrm{mL}$ ) was injected into the caudal epidural space in accordance with our clinical protocol.

In the fluoroscopy-guided group (Group F), the patients were placed in the prone position and the caudal epidural space was visualized with the C-arm (Ziehm Imaging, Nünberg, Germany) in the lateral position. CESI was not performed in patients with anomalies in the sacral and caudal structures. After subcutaneous local anesthetic infiltration at the injection site, the caudal block needle was advanced into the caudal epidural space with intermittent fluoroscopy imaging (Figure 2A). After determining negative blood aspiration and CSF, the epidural space was confirmed in the lateral and anteroposterior positions with $1 \mathrm{~mL}$ of radiopaque material (Figures $2 B, 2 \mathrm{C}$ ). Similar to Group $\mathrm{U}$, a $10-\mathrm{mL}$ mixture of $2 \mathrm{~mL}$ of 8 $\mathrm{mg}$ dexamethasone and $2 \mathrm{~mL}$ of $2 \%$ lidocaine in saline was injected into the caudal epidural space.

After the data were transferred to the computer, the analysis was performed using SPSS 21.0 software (SPSS, Chicago, IL, USA). Descriptive statistics are presented as numbers (percentages) and means and standard deviation. The Kolmogorov-Smirnov test was performed to determine whether the continuous data followed normal distribution. Continuous data with normal distribution in the dependent groups were compared using the Friedman test and those in the independent groups were compared using the t-test. The Fisher's exact test was performed to compare the discrete data of individual groups. Statistical significance was set at $\mathrm{p}<0.05$.

\section{RESULTS}

This study included 180 patients (Group U: 90 patients, Group F: 90 patients). Demographic data and procedure times for both the groups are presented in Table I. The procedure time was significantly lower in Group $U$ than in Group F $(p=0.001)$.
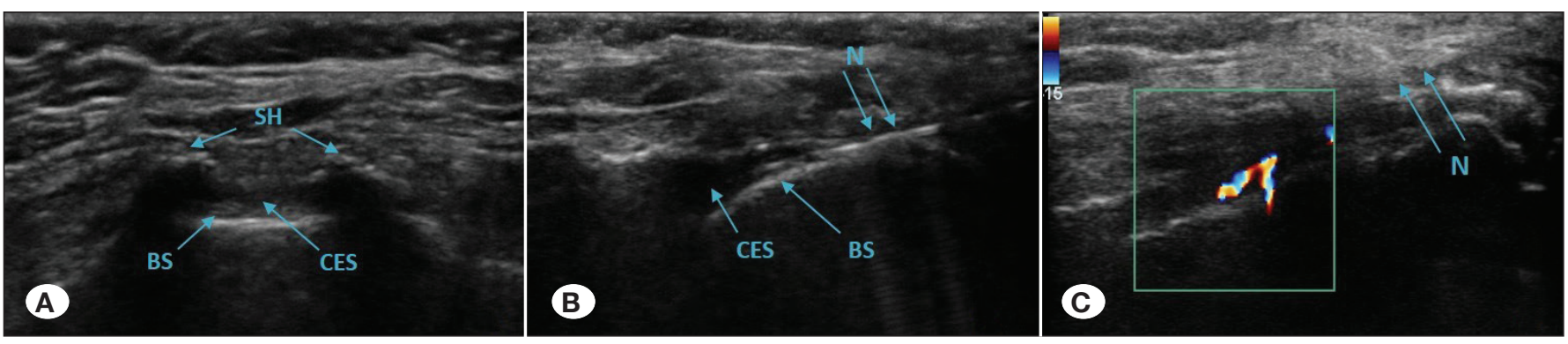

Figure 1: Sacral hiatus ultrasound images indicating the postion of the needle in the caudal epidural space. A) Transverse view. B) Longitudinal view. The position of the needle in the caudal epidural space (CES: Caudal Epidural Space, BS: Base of Sacrum, SH: Sacral Horn, N: Needle). C) Distribution of the injected solution with color Doppler ultrasound. 
In addition, the successful injection rate on the first attempt was significantly higher in Group $U$ than in Group F ( $p=0.002)$.

The difference between the groups for the number of subcutaneous injections and the rate of blood and CSF aspiration were statistically insignificant (Table II).

The NRS scores of the patients before and at 3 weeks and 3 months after the injection are presented in Table III. The differences in the NRS scores of the two groups before and at 3 weeks and 3 months after the injection were insignificant $(p=0.36,0.22,0.47$, respectively). However, statistically significant differences were noted between the preoperative and postoperative (at the third week and the third month) NRS scores in both the groups $(p<0.001)$.

The ODI scores of the patients before and at 3 weeks and 3 months after the injection are presented in Table IV. The differences in the ODI scores of both the groups before and at 3 weeks and 3 months after the injection were statistically insignificant ( $p=0.57,0.58,0.22$, respectively). Statistically significant differences were noted between the preoperative and postoperative (at the third week and the third month) ODI scores in both the groups $(p<0.001)$.

\section{DISCUSSION}

The current study assessed the efficacy of CESI under US and fluoroscopy guidance and demonstrated significant improvement in the NRS and ODI scores at mid-term followup, without significant differences between the two groups. However, US-guided CESI had superior outcomes considering the procedure time and successful injection rate on the first attempt. Notably, US guidance has advantages such as no exposure to radiation.

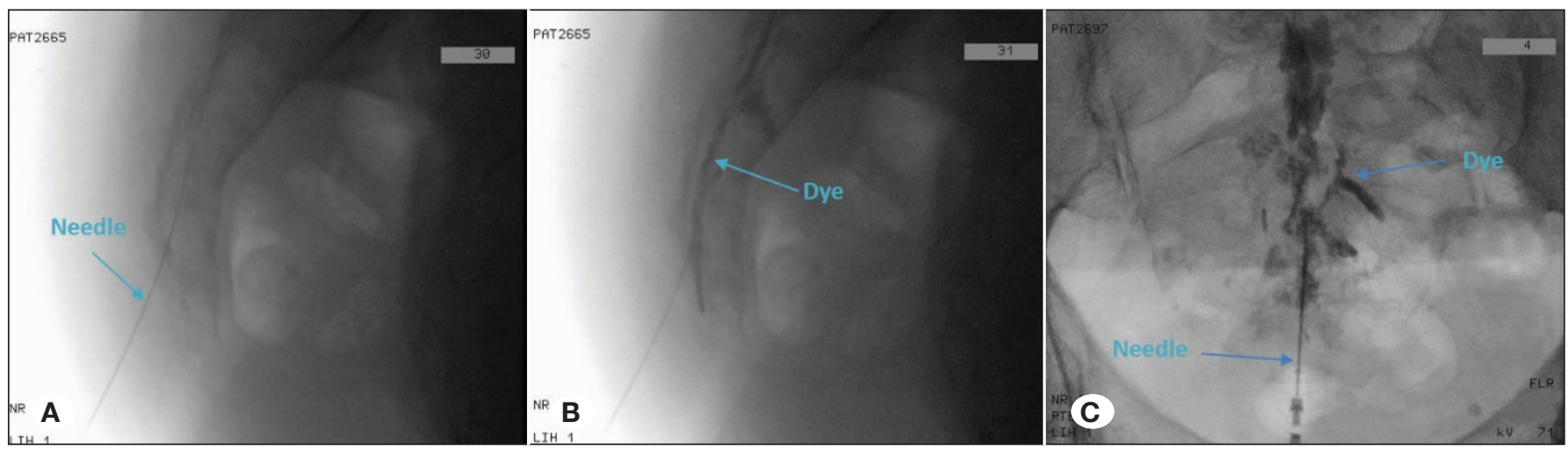

Figure 2: Fluoroscopic images caudal epidural injection. A) Lateral view of injection needle. B) Lateral view of caudal injection with dye. C) Antero-posterior view of caudal injection with dye.

Table I: General Characteristics of the Patients

\begin{tabular}{lccc}
\hline & Group U (n=90) & Group F (n=90) & p \\
\hline Age (mean \pm SD), years & $63.34 \pm 9.57$ & $61.96 \pm 9.12$ & 0.32 \\
\hline Weight (mean \pm SD), kg & $79.92 \pm 13.28$ & $84.17 \pm 11.98$ & $\mathbf{0 . 0 2}$ \\
\hline Height (mean \pm SD), cm & $165.22 \pm 7.76$ & $167.36 \pm 8.78$ & 0.30 \\
\hline BMl (mean \pm SD) & $29.44 \pm 5.38$ & $30.57 \pm 6.23$ & 0.19 \\
\hline Procedure Time (mean \pm SD), minutes & $6.46 \pm 1.84$ & $10.11 \pm 3.65$ & $<\mathbf{0 0 1}$ \\
\hline
\end{tabular}

BMI: Body Mass Index.

Table II: Invariable Analysis for Possible Outcome Predictors for Injection Effectiveness at Follow-Up Period

\begin{tabular}{lccc}
\hline & Group U (n=90) & Group F (n=90) & p \\
\hline Successful Injection on First Attempt; $n(\%)$ & $85(94.4)$ & $70(77.7)$ & $\mathbf{0 . 0 0 2}$ \\
\hline Complications & & & $6(6.6)$ \\
$\quad$ Subcutaneous Injections; $n(\%)$ & $6(6.6)$ & $16(17.8)$ & 1 \\
Blood Aspiration; $n(\%)$ & $14(15.6)$ & $2(2.3)$ & 0.84 \\
CSF Aspiration; $n(\%)$ & $3(3.4)$ & 0.92 \\
\hline
\end{tabular}

CSF: Cerebrospinal fluid. 
Senkal S. and Sir E: Caudal Epidural Steroid Injection

Table III: Numeric Rating Scale (NRS) Scores of the Patients Before, and After the Treatment

\begin{tabular}{|c|c|c|c|c|c|}
\hline NRS & Group U (n=90) & Group F (n=90) & $\mathbf{p}^{*}$ & $\mathbf{p}^{* *}$ & $\mathbf{p}^{\star * *}$ \\
\hline Post-Injection; $3^{\text {rd }}$-week & $2.68 \pm 1.14$ & $2.48 \pm 1.05$ & $<0.001$ & $<0.001$ & 0.22 \\
\hline Post-Injection; $3^{\text {rd }}$-month & $2.72 \pm 0.84$ & $2.82 \pm 1.01$ & $<0.001$ & $<0.001$ & 0.47 \\
\hline
\end{tabular}

* Significance of the group $U$ from pre-procedure value. ** Significance of the group $F$ from pre-procedure. *** Significance between the group $U$ and the group $F$.

Table IV: Oswestry Disability Index (ODI) Scores of the Patients Before, and After the Treatment

\begin{tabular}{|c|c|c|c|c|c|}
\hline ODI & Group U $(n=90)$ & Group F $(n=90)$ & $\mathbf{p}^{*}$ & $\mathbf{p}^{\star *}$ & $\mathbf{p}^{\star \star *}$ \\
\hline Post-Injection; $3^{\text {rd }}$-week & $33.67 \pm 13.09$ & $32.75 \pm 8.91$ & $<0.001$ & $<0.001$ & 0.58 \\
\hline Post-Injection; $3^{\text {rd }}$-month & $31.57 \pm 8.78$ & $33.35 \pm 10.67$ & $<0.001$ & $<0.001$ & 0.22 \\
\hline
\end{tabular}

* Significance of the group $U$ from pre-procedure value. ${ }^{* *}$ Significance of the group $F$ from pre-procedure. ${ }^{* * *}$ Significance between the group $U$ and the group $F$.

CESI can be administered with blind, fluoroscopy-, or US-guided techniques $(11,17)$. Blind epidural injection by palpating the sacral horns to confirm the hiatus without radiological guidance is associated with complications such as subcutaneous, intraosseous, intrathecal, and intravascular injections and an incidence rate of $14 \%-56 \%$ for needle misplacement (3). Fluoroscopy and US guidance lower these adverse events and complications (8). However, fluoroscopy exposes patients and physicians to a considerable amount of radiation doses (20). Hence, we performed US-guided CESI and found that US-guided CESI had a lower complication rate than fluoroscopy-guided technique as well as the blind technique, which was performed in previous studies (10).

In fluoroscopy-guided injections, the needle is advanced by performing intermittent imaging. In addition, because 2D imaging is performed, the needle may sometimes appear to be at correct location even when the needle is not at the correct location. Hence, only if images are obtained from different angles, the incorrect position of the needle can be confirmed. Consequently, the needle is required to be retracted and repositioned in these patients. However, real-time and 3D imaging is performed in US-guided injections. This provides immediate confirmation of the needle location and prevents time-wasting because of continuous imaging during the advancement of the needle. In a prospective randomized study comparing US- and fluoroscopy-guided approaches in 30 patients with post-laminectomy syndrome, Akkaya et al. found that the procedure time was shorter in the US group (1). Similarly, the procedure time was found to be shorter in Group $U$ than in Group $F$ in the current study.

Recently, many studies have been conducted on anatomical and morphometric features and variations relevant to caudal epidural injection (10). Comprehensive knowledge of the anatomy of the region increases the success rate of the procedure and reduces the risk of complications. Sekiguchi et al. examined 92 sacrum specimens and reported that the sacral hiatus was closed in 3 specimens and the caudal block failure rate was higher in patients with sacral canal diameter less than $2 \mathrm{~mm}$ (19). Similarly, in the current study, the sacral hiatus was closed in 1 patient in Group $U$ and in 2 patients in Group F. Sacral hiatus patency can be easily assessed with both fluoroscopy and US. However, while only bony structures can be observed on fluoroscopy, adjacent soft tissues, vulnerable vessels, the sacrococcygeal ligament, sacral hiatus, and sacral canal can be imaged with US in real time. Thus, the injection can be performed with a higher success rate in less time under US guidance. Therefore, this may be the reason for the higher successful injection rate on the first attempt in Group $U$ in this study.

Although the intravascular distribution in fluoroscopy-guided caudal block can be easily detected by injecting radiopaque material, Fukazawa et al. reported only $11 \%-42 \%$ of intravascular injections are performed under fluoroscopy guidance (7). On the other hand, no reliable markers such as a radiopaque dye are available for US-guided caudal injections. However, the expansion and turbulence in the sacral canal confirm that the injection was made to the correct site. Injection of fluid into the caudal epidural space causes turbulent flow, which appears as a burst of color, whereas intrathecal injection does not appear as a burst of color in Doppler mode (1). Although US seems to be more advantageous, no significant difference was observed between the techniques considering the rate of complications such as intravascular, intrathecal, intraosseous, and subcutaneous injections.

This study has some limitations. To better understand the causes of complaints of patients, clinical data and imaging details such as quantitative analyses of disc herniations, canal diameters, degree of disc degeneration, and facet degeneration should be assessed. In addition, because this was a retrospective study, patients were not randomized during 
the procedure. Hence, the patients in Group $U$ were weaker, which may have resulted in the higher successful injection rate on the first attempt in Group U. Moreover, although the adverse effects of radiation exposure were reported in Group $\mathrm{F}$, the exposure dose to each patient and physician were not recorded. Finally, the 3-month follow-up period is insufficient for comparing the medium- and long-term efficacies of both the techniques. Therefore, large-scale prospective studies with long-term follow-up investigating the superiority of USguided CESI over fluoroscopy-guided CESI are warranted.

\section{CONCLUSION}

US-guided CESI had a shorter procedure time and higher successful injection rate on the first attempt than fluoroscopyguided CESI, whereas pain relief and functional improvement were similar in both techniques. Therefore, we believe that USguided CESI should be considered as an effective alternative for pain management in patients with chronic LBP.

\section{REFERENCES}

1. Akkaya T, Ozkan D, Kertmen H, Sekerci Z: Caudal epidural steroid injections in postlaminectomy patients: Comparison of ultrasonography and fluoroscopy. Turk Neurosurg 27(3):420425, 2017

2. Alexander CE, Varacallo M: Lumbosacral radiculopathy. In: StatPearls [Internet]. Treasure Island (FL): StatPearls Publishing; 2020 Jan. Available at: https://pubmed.ncbi.nlm. nih.gov/28613587/ Accessed Mar 25, 2020

3. Blanchais A, Le Goff B, Guillot P, Berthelot JM, Glemarec J, Maugars Y: Feasibility and safety of ultrasound-guided caudal epidural glucocorticoid injections. Joint Bone Spine 77(5):440444, 2010

4. Chang-Chien GC, Knezevic NN, McCormick Z, Chu SK, Trescot AM, Candido KD: Transforaminal versus interlaminar approaches to epidural steroid injections: A systematic review of comparative studies for lumbosacral radicular pain. Pain Physician 17(4):E509-524, 2014

5. Chou R, Hashimoto R, Friedly J, Fu R, Dana T, Sullivan S, Bougatsos C, Jarvik J: Pain Management Injection Therapies for Low Back Pain. Rockville (MD): Agency for Healthcare Research and Quality (US), 2015

6. Chou R, Qaseem A, Snow V, Casey D, Cross JT, Shekelle P, Owens DK: Clinical Efficacy Assessment Subcommittee of the American College of Physicians, American College of Physicians, American Pain Society Low Back Pain Guidelines Panel. Diagnosis and treatment of low back pain: A joint clinical practice guideline from the American College of Physicians and the American Pain Society. Ann Intern Med 147(7):478-491, 2007
7. Fukazawa K, Matsuki Y, Ueno H, Hosokawa T, Hirose M: Risk factors related to accidental intravascular injection during caudal anesthesia. J Anesth 28(6):940-943, 2014

8. Hazra AK, Bhattacharya D, Mukherjee S, Ghosh S, Mitra M, Mandal M: Ultrasound versus fluoroscopy-guided caudal epidural steroid injection for the treatment of chronic low back pain with radiculopathy: A randomised, controlled clinical trial. Indian J Anaesth 60(6):388-392, 2016

9. Hsieh LLC, Kuo CH, Lee LH, Yen AMF, Chien KL, Chen THH: Treatment of low back pain by acupressure and physical therapy: Randomised controlled trial. BMJ 332(7543):696700, 2006

10. Kao SC, Lin CS: Caudal epidural block: An updated review of anatomy and techniques. Biomed Res Int 2017:9217145, 2017

11. Klocke R, Jenkinson T, Glew D: Sonographically guided caudal epidural steroid injections. J Ultrasound Med 22(11):12291232, 2003

12. Liu J, Zhou H, Lu L, Li X, Jia J, Shi Z, Yao X, Wu Q, Feng S: The effectiveness of transforaminal versus caudal routes for epidural steroid injections in managing lumbosacral radicular pain: A systematic review and meta-analysis. Medicine (Baltimore) 95(18):e3373, 2016

13. Manchikanti L, Cash KA, McManus CD, Pampati V: Fluoroscopic caudal epidural injections in managing chronic axial low back pain without disc herniation, radiculitis, or facet joint pain. J Pain Res 5:381-390, 2012

14. Marshall LL, Trethewie ER, Curtain CC: Chemical radiculitis. A clinical, physiological and immunological study. Clin Orthop Relat Res (129):61-67, 1977

15. Messina C, Orlandi D, Sconfienza LM: Do we still need fluoroscopy to perform injections in the musculoskeletal system? Skeletal Radiol 45(12):1717-1718, 2016

16. Ogoke BA: Caudal epidural steroid injections. Pain Physician 3(3):305-312, 2000

17. Park Y, Lee JH, Park KD, Ahn JK, Park J, Jee H: Ultrasoundguided vs. fluoroscopy-guided caudal epidural steroid injection for the treatment of unilateral lower lumbar radicular pain: A prospective, randomized, single-blind clinical study. Am J Phys Med Rehabil 92(7):575-586, 2013

18. Patel K, Chopra P, Upadhyayula S: Epidural Steroid Injections. In: StatPearls Article Knowledge Base website]. Treasure Island (FL): StatPearls Publishing, 2020 Available at: https:// www.statpearls.com/kb/viewarticle/21232 Accessed April 7, 2020

19. Sekiguchi M, Yabuki S, Satoh K, Kikuchi S: An anatomic study of the sacral hiatus: A basis for successful caudal epidural block. Clin J Pain 20(1):51-54, 2004

20. Vakil C: Radiation and medical procedures: How do we do no harm? Can Fam Physician 63(10):774-775, 2017 